\title{
ON \\ O PESO REAL Do Esôfago \\ DE BARRETT
}

\section{Autores:}

\section{Heloisa Novaes}

PhD pela Fundação Osvaldo Cruz (Fiocruz)

Mestrado pela Universidade Federal do Rio de Janeiro

Patologista do Instituto Fernandes Figueira, Fiocruz

\section{Glaciomar Machado}

Professor Titular de Gastroenterologia da Faculdade de Medicina da Universidade Federal do Rio de Janeiro

$\mathrm{PhD}$ pela Universidade de Bristol, Inglaterra

Membro-Titular, Academia Nacional de Medicina

(cadeira 18)

Presidente-honorário, World Endoscopy

Organization - WEO/OMED (2005-)

A incidência de adenocarcinoma do esôfago aumentou 500\% desde 1970, valor este superior ao de qualquer outro câncer no mundo ocidental (Pohl H; Welch HG, 2005), com taxas de sobrevida de 5 (cinco) anos ainda consideradas baixas, entre 15\% - 20\% (Wani S et al., 2011), apesar dos progressos recentes, tanto no diagnóstico quanto na conduta desta entidade letal.

Considerado uma das condiçôes prémalignas para o desenvolvimento do adenocarcinoma esofagiano, o esôfago de Barrett tem risco anual para progredir para displasia de alto grau/adenocarcinoma estimado em 0,58\% - 0,63\% (Wani S et al., 2011).
O esôfago de Barrett (descrito inicialmente por Norman Barrett - Barrett NR, 1957) é hoje definido como uma anormalidade metaplásica adquirida na qual qualquer extensão de epitélio escamoso estratificado do esôfago é substituída por epitélio colunar semelhante ao do intestino contendo, obrigatoriamente, células caliciformes (metaplasia intestinal), que podem ser mais bem evidenciadas quando coradas pelo PAS com alcian-blue (Spechler SJ; Souza RF, 2014).

Neste capítulo iremos discutir e apresentar a real posição do esôfago de Barrett no desenvolvimento do adenocarcinoma esofagiano.

\section{História Natural}

Do ponto de vista de sua história natural, a progressão do esôfago de Barrett para adenocarcinoma é processo gradativo (Quadro 1).

Pesquisas realizadas em animais por Thiesen et al. em 2003 demonstraram, categoricamente, que a doença do refluxo gastroesofagiano, em pacientes com hérnia hi- 
atal por deslizamento, é pré-requisito fundamental para o desenvolvimento do adenocarcinoma esofagiano: é que a mucosa do esôfago, agredida pelo refluxo gastroduodenal, desenvolve, ao longo do tempo, esofagite crônica e, na tentativa de se defender dessa agressão, é substituída por epitélio metaplásico, sem displasia. Em sequência, e de forma gradativa, o epitélio metaplásico pode apresentar displasia de baixo grau (DBG), evoluir sucessivamente para displasia de alto

\section{Prevalência}

Apesar de a elevação da incidência do adenocarcinoma do esôfago ser inquestionável, ainda existe alguma dúvida quanto ao aumento da incidência do esôfago de Barrett na população ao longo do tempo. Tal incerteza se justifica não apenas por ser o Barrett frequentemente assintomático (cerca de 6\% dos pacientes - Modiano N; Gerson LB, 2007), mas também pela

\section{Esôfago de Barrett: História Natural}

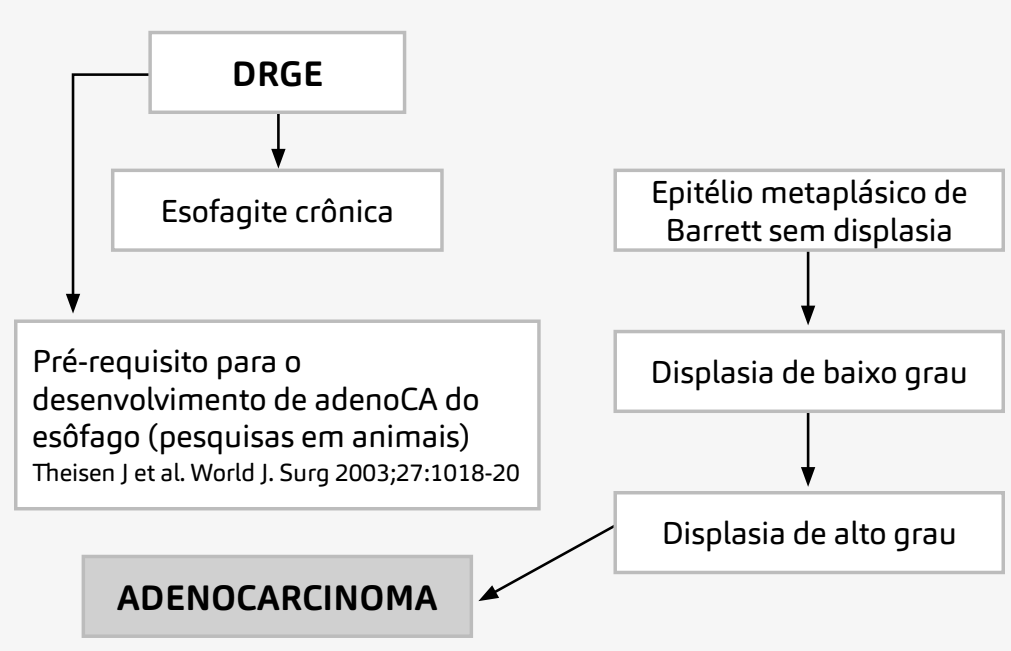

grau (DAG), adenocarcinoma intramucoso e adenocarcinoma invasivo (Spechler SJ et al., 2018). necessidade do emprego de procedimentos especializados para o estabelecimento de seu diagnóstico - endoscopia digestiva 
alta e confirmação histopatológica (ASGE Standards of Practice Committee, 2012) porém o acesso à endoscopia está longe de ser universal, mesmo considerando os pacientes que apresentam sintomatologia. Portanto, a análise das endoscopias ou da histopatologia de hospitais qualificados não representa a verdadeira prevalência do esôfago de Barrett. Além disto, os casos identificados por estes exames provavelmente diferem em termos de características demográficas e mesmo de saúde dos indivíduos que vivem com esôfago de Barrett não diagnosticado. Levando-se em consideraçáo estas limitaçóes, os dados epidemiológicos mostram que a doença do refluxo gastroesofagiano é um importante fator de risco para o desenvolvimento da metaplasia de Barrett, que ocorre em $6 \%$ $14 \%$ destes pacientes, predominantemente no sexo masculino na proporção de 2:1, cujo diagnóstico foi estabelecido geralmente entre 50-59 anos de idade. Em relação a outros fatores de risco, os estudos sugerem prevalência maior nos brancos e idosos, tabagismo, alimentação insuficiente em frutas e vegetais e aumento de extensão do epitélio metaplásico (Steevens J et al., 2010; Coleman HG et al., 2012; Hampel H et al., 2005).

Para contornar estas limitaçóes, investigadores realizaram, sistematicamente, endoscopia digestiva alta em pacientes encaminhados para se submeterem a outros exames. Os números encontrados sugerem que a maioria de casos de esôfago de Barrett não é detectada na população em geral. Este fato encontra respaldo em estudo mais recente, que indica que $95 \%$ a $98 \%$ dos pacientes com esôfago de Barrett permanecem sem identificação e que a prevalência do esôfago de Barrett em indivíduos sem sintomatologia de refluxo situa-se entre $0,4 \%$ e $6 \%$ (Wang K, 2008).

\section{Carcinogênese}

O esôfago de Barrett é clinicamente muito importante por ser considerado o principal fator de risco do adenocarcinoma do esôfago, cuja incidência foi a que cresceu mais rapidamente entre todos os tumores, sendo estimada em $0,58 \%$ - 0,63\%. Este valor, considerado pequeno em termos absolutos, corresponde, na realidade, a um aumento na incidência 40 a 100 vezes maior que o encontrado na população geral (Pohl $\mathrm{H}$; Welch HG, 2005).

Uma vez estabelecido o diagnóstico de epitélio metaplásico de Barrett, o risco de progressão para câncer é imprevisível e as estratégias atuais para acompanhamento são desapontadoras para o clínico e para o paciente, além de aparentemente não alterar a morbidade e mortalidade do câncer de esôfago. É, portanto, necessária uma melhor compreensáo dos fatores genéticos e ambientais no desenvolvimento e progressão do 
esôfago de Barrett para diminuir o crescimento exponencial do adenocarcinoma e para melhorar os resultados das diversas formas de tratamento disponíveis.

Aceita-se que a carcinogênese no esôfago de Barrett tenha a seguinte sequência: metaplasia intestinal, displasia de baixo grau, displasia de alto grau, adenocarcinoma intramucoso e finalmente adenocarcinoma invasivo (Spechler SJ et al., 2018).

\section{Conduta Clínica}

O esôfago de Barrett é o fator de risco mais importante para o desenvolvimento do adenocarcinoma esofagiano que, no estágio avançado, tem taxa de mortalidade de aproximadamente $90 \%$ (Wild CP, Hardie LJ, 2003). Portanto, a detecção precoce do câncer ou a prevenção da progressão indesejável do esôfago de Barrett são estratégias óbvias que devem ser consideradas na conduta clínica desta entidade. As opçôes mais utilizadas são: ou o tratamento do refluxo gastroesofagiano e controle do risco de desenvolvimento de câncer, através de acompanhamento por endoscopia com biópsias seriadas, indicando-se a esofagectomia nos casos que apresentem displasia de alto grau e/ou carcinoma intramucoso, pré-invasivo, ou o emprego de técnicas de ablação endoscópica.

A maioria das normas internacionais (Spechler SJ et al., 2011; Wang K, Sampliner
$\mathrm{R}$, 2008) recomenda o acompanhamento endoscópico com biópsias nos casos de Barrett sem displasia a cada 3-5 anos com o objetivo de detectar sinais precoces de displasia de alto grau ou de câncer ainda em fase curável. Justifica-se tal conduta considerando-se que, se a progressão para displasia de baixo grau, displasia de alto grau ou adenocarcinoma puder ser detectada precocemente, o câncer pode ser tratado em estágio superficial, curável (Spechler SJ et al., 2011; Prasad GA et al., 2010). Entretanto, há que se considerar o trabalho prospectivo conduzido sob o patrocínio do American College of Gastroenterology que indica que o adenocarcinoma se instala em cerca de $18 \%$ dos pacientes com esôfago de Barrett e displasia de baixo grau e em até $34 \%$ naqueles com displasia de alto grau (Shaheen NJ, Falk GW, Iyer PG et al., 2016). Portanto, a displasia representa um difícil dilema terapêutico: ou o acompanhamento endoscópico para detecçáo de possível progressão para displasia de alto grau $\mathrm{e}$ câncer limitado à mucosa, ou esofagectomia.

Um número considerável de Serviços recomenda acompanhar os pacientes com displasia de baixo grau, considerada como lesão pré-cancerosa, com endoscopia e biópsias múltiplas anualmente; caso persista a displasia de baixo grau por 3 (três) anos consecutivos, este intervalo passa a ser de 3/3 anos. Entretanto, nos últimos anos, a terapia endoscópica tem sido cada vez mais 
recomendada nestes pacientes, considerando a gama de recursos terapêuticos que a endoscopia oferece, como veremos no tópico seguinte (Bennett $C$ et al., 2015).

A esofagectomia, por outro lado, empregada como tratamento de escolha para os pacientes com esôfago de Barrett e displasia de alto grau ou adenocarcinoma, tem sido reservada, no momento, para os casos em que o adenocarcinoma ultrapassa a camada mucosa, invadindo as demais camadas. A recomendaçáo atual é tratar displasia de alto grau e o adenocarcinoma limitado à mucosa com as novas terapêuticas endoscópicas, como ablação e mucosectomia, por serem efetivas, por preservarem o esôfago e pelo fato de que a esofagectomia cursa com índices significativos de morbidade e mortalidade (morbidade entre $18 \%$ a $48 \%$ e mortalidade de 3\% a 5\%) (Bennett C et al., 2012).

\section{O Tratamento Endoscópico Do Esôfago de BARRETT}

O conceito da prevenção do adenocarcinoma associado ao esôfago de Barrett por erradicação do epitélio metaplásico prémaligno surgiu no início dos anos $1990 \mathrm{com}$ dois trabalhos que derrubaram o dogma segundo o qual o esôfago de Barrett, uma vez formado, torna-se uma lesão irreversível. Berenson (Berenson MM, 1998) e Sampliner e cols. (Sampliner R et al., 1996) demons- traram, independentemente, a reversão inequívoca do epitélio de Barrett sem displasia, com restauração do epitélio escamoso esofagiano normal, ao produzirem ablação térmica do tecido metaplásico e sua cicatrização, associando potentes inibidores da secreçáo ácida do estômago. Assim, nasceu a noção de que é possível eliminar total ou parcialmente a metaplasia intestinal, principal fator de desenvolvimento do câncer de esôfago.

Sabe-se que a invasão da submucosa pelo adenocarcinoma, originário do epitélio metaplásico, tem $25 \%$ de risco de desenvolvimento de metástases para os linfonodos (Bergman JJ, 2018). Como a metaplasia é processo que atinge apenas a mucosa, o câncer limitado à mucosa e a metaplasia de Barrett com ou sem displasia são os candidatos apropriados para as diferentes formas de terapêutica endoscópica (Sampliner RE, 1997).

Uma vez estabelecido o diagnóstico de esôfago de Barrett, a conduta a seguir depende da presença e do grau de displasia. Nos pacientes com displasia de alto grau, as evidências apontam que a erradicação endoscópica está associada à diminuição do risco de progressão para adenocarcinoma, quando comparada com os pacientes submetidos, apenas, ao acompanhamento endoscópico (Prasad GA et al., 2007). Existem também evidências de que a erradicação de displasia de baixo grau resulta numa taxa menor de progressão para displasia de alto grau ou adenocarcinoma (Phoa KN et al., 
2014). No momento, entretanto, não há unanimidade quanto à conduta nos pacientes com Barrett e displasia de baixo grau: enquanto o Colégio Americano de Gastroenterologia (ACG) e a Associação Americana de Gastroenterologia (AGA) recomendam a erradicação endoscópica (Shahen NJ et al., 2016; Spechler SJ et al., 2011), a Sociedade Britânica de Gastroenterologia (BSG) sugere o acompanhamento endoscópico (Fitzgerald RC et al., 2014). Quanto aos pacientes com esôfago de Barrett sem displasia, a erradicação endoscópica de rotina não é recomendada atualmente em razão do baixo risco de sua progressão para neoplasia (Wani $S$ et al., 2011); o risco pequeno, porém real, de complicaçóes e os custos inerentes ao tratamento endoscópico (Hur C et al., 2012).

Os métodos endoscópicos de ablação do esôfago de Barrett, disponíveis no Brasil, mais empregados na atualidade e considerados, a nível internacional, os mais eficazes para o tratamento do epitélio metaplásico, são (Machado G, 2016):

(1) eletrocoagulação com plasma de Argônio (APC);

(2) ablação por radiofrequência;

(3) mucosectomia;

(4) a dissecção submucosa;

(5) a combinação destes métodos.

Ao dispormos das alternativas endoscópicas aqui referidas, para tratar o esôfago de Barrett, deparamo-nos com o seguinte di- lema: como decidir quando fulgurar, quando ressecar ou quando empregar ambos? David Fleisher analisa, com rara lucidez, os diferentes métodos endoscópicos de tratamento (Fleischer DE, 2012) e sugere que, para a tomada de tal decisão, sejam considerados os seguintes parâmetros: (1) a macroscopia da lesão; (2) as suas dimensões; (3) se é circunferencial ou não e (4) a profundidade do processo patológico na parede do esôfago.

Embora os métodos referidos variem quanto à resposta que produzem à destruição da mucosa, parece que esta destruição, num meio livre de ácido (obtido pela associação de potentes bloqueadores da bomba de prótons), é geralmente seguida de regeneração com mucosa escamosa histologicamente normal (Singh S et al., 2014). Estudos randomizados suportam a hipótese de que a ablação possa prevenir a malignizaçáo do Barrett, porém não descartam a possibilidade potencial de malignização de áreas remanescentes de mucosa colunar e até mesmo de mucosa escamosa neoformada (Van Laethem JL et al., 2000).

Se a ablação reduzisse o risco de desenvolvimento de câncer e seu custo-benefício fosse aceitável, o paradigma atual de acompanhamento endoscópico poderia ser direcionado para triagem, seguida de ablação de todos os esôfagos de Barrett porventura identificados. Contudo, até o momento, não existem evidências que suportem esta estratégia. 
A crioterapia não será discutida no momento, por ainda não ser empregada rotineiramente e por serem os resultados obtidos, embora promissores (Ghorbani $S$ et al., 2016), insuficientes em número (Spechler SJ, 2018).

Comentaremos, portanto, os métodos endoscópicos mais empregados na atualidade e considerados os mais eficazes para o tratamento do epitélio metaplásico.

\section{Eletrocoagulação com plasma de argônio (APC)}

A eletrocoagulação (eletrofulguração) com plasma de argônio (APC) emprega a eletrocoagulação monopolar através da qual uma corrente de alta frequência é aplicada ao tecido, por meio de um gás ionizado, eletricamente condutor (plasma de argônio), portanto, sem a necessidade de contato com o tecido. Devido ao fluxo divergente do gás, esta corrente é transmitida tanto lateral, quanto axialmente. Dependendo do fluxo de gás e da força do campo elétrico, obtémse coagulação térmica do tecido por uma profundidade de 2 a 4 milímetros (Machado G, Novaes H, 2001). Neste ponto, levantase uma questão: esta profundidade seria suficiente para a ablação de todo o epitélio metaplásico? Fleischer (Fleischer DE, 2012) responde a esta indagação positivamente, uma vez que a eletrofulguração com plasma de argônio atinge mucosa e submucosa, enquanto o epitélio esofagiano normal mede cerca de 0,5 mm e o epitélio de Barrett, 0,6 $\mathrm{mm}$, estendendo-se, portanto, à parte da submucosa (mede $0,5 \mathrm{~mm}$ ).

Os resultados obtidos com a eletrocoagulação com plasma de argônio (APC) da maioria dos artigos publicados nos últimos anos (excluídos os referentes à apresentação de casos isolados e/ou resumos de trabalhos apresentados em congressos) são efetivos para a erradicação do epitélio metaplásico de Barrett (Machado G, Novaes H, 2001).

Temos tido resultados positivos com o emprego deste método, sendo que atribuímos nosso sucesso aos seguintes fatores:

- Ao contrário de grande parte dos autores, preferimos realizar o tratamento em várias sessóes, cada uma delas restrita a um quadrante do esôfago, por uma extensão de, no máximo, $5 \mathrm{~cm}$. No esôfago de Barrett longo $(>5 \mathrm{~cm})$, iniciamos o tratamento pela porção distal do órgão, até completar a totalidade de sua circunferência, momento em que passamos a tratar a metaplasia a partir de $5 \mathrm{~cm}$ (tratados inicialmente) em sentido proximal, cada sessão compreendendo 1/4 do diâmetro do esôfago. Com esta rotina reduzimos a níveis quase nulos a incidência de estenose pós-tratamento.

- Utilizamos a potência de $80 \mathrm{~W}$ em nossos pacientes, ao contrário de $60 \mathrm{~W}$. Desta forma, atingimos a submucosa do esôfago e, consequentemente, o surgimento de células caliciformes. 


\section{Ablaçáo por radiofrequência (ARF)}

Este método emprega um baláo cilíndrico que, ao ser insuflado, coloca múltiplos eletródios em contato com a mucosa esofagiana, liberando energia por radiofrequência (Sharma VK et al., 2007). Esta modalidade de tratamento é altamente efetiva para a erradicação de displasia nos pacientes com esôfago de Barrett, alcançando $81 \%$ nas displasias de alto grau e $91 \%$ nas de baixo grau (Sharma VK et al., 2009) e a erradicação completa decorridos 12 meses em ensaio randomizado controlado com placebo (Shaheen NJ et al., 2009). Estes últimos autores observaram também que houve redução do risco de progressão para adenocarcinoma nos pacientes com displasia (de baixo alto e de alto grau) nos quais a progressáo para displasia de alto grau ou câncer do que naqueles submetidos à terapêutica com placebo ( $4 \%$ versus $16 \%$ ). Em uma metanálise de 20 estudos, a erradicação completa de displasia foi obtida em 91\% dos pacientes (Orman ES, Li N, Shaheen NJ, 2013). Embora os estudos iniciais sugerissem que as recorrências fossem incomuns, trabalhos mais recentes têm documentado taxas consideráveis de recorrência. Por exemplo, Small AJ et al. (2015) trataram 246 pacientes com displasia de alto grau ou carcinoma intramucoso, obtendo, inicialmente, completa erradicaçáo da metaplasia intestinal em $80 \%$ dos casos. Entretando, houve recorrência de displasia ou câncer em aproximadamente $25 \%$ decorridos 60 meses, e de metaplasia intestinal em cerca de $50 \%$ aos 48 meses.

Embora a ablação por radiofrequência seja o método mais empregado atualmente nos Estados Uniddos da América e o que apresenta resultados mais eficazes para o tratamento do esôfago do Barrett, sua utilização em nosso meio tem restriçóes em virtude de seu alto custo, não somente relacionado à aparelhagem mas, também, à manutençáo do equipamento que requer técnicos bem qualificados e especializados no manuseio deste material.

Desde junho de 2018 estamos utilizando a ablação por radiofrequência em nosso serviço com bons resultados, embora nossa casuística ainda seja pequena para conclusóes definitivas. Entretanto, considerando os resultados que temos obtido com o plasma de argônio, no momento nossa preferência recai sobre este método para a ablação do epitélio metaplásico.

\section{Mucosectomia (EMR)}

A ressecção endoscópica inclui a mucosectomia e a dissecção submucosa e envolve a excisão de um extenso segmento de mucosa até a submucosa (Soetikno RM et al., 2002). Foi utilizada inicialmente para o tratamento de pacientes com carcinoma do esôfago, estômago ou do cólon, limitado à mucosa (estágio T1m1-3) (Spechler SJ, August 3, 2018). 
Ao contrário dos métodos endoscópicos comentados anteriormente, a ressecção endoscópica é o único método que permite a remoçáo do tecido e, consequentemente, o diagnóstico histopatológico definitivo. Considerando que o exame da peça pode diferir do diagnóstico histopatológico das biópsias prévias e/ou do estadiamento da lesão por endossonografia do esôfago, este fato é de particular importância na seleção da melhor terapêutica a ser recomendada (Ell C et al., 2000).

As complicações inerentes à mucosectomia são, em geral, pouco frequentes, embora a estenose seja comum nos casos em que extensas áreas de mucosa são ressecadas (Ell C et al., 2000; Lewis JJ et al., 2011). $\mathrm{O}$ tratamento de escolha é a dilatação por via endoscópica (Katada C et al., 2003). Outras complicaçóes incluem hemorragia, usualmente tratada endoscopicamente (Ell C et al., 2000; Chennat J et al., 2009) e perfuração ( $<1 \%$ a $5 \%$ ) (Pech $\mathrm{O}$ et al., 2014). O risco de complicaçôes aumenta na proporção do número de ressecçóes realizadas. Por esta razáo, a mucosectomia da totalidade de mucosa metaplásica de Barrett é geralmente limitada a segmentos curtos (Eluri S; Shaheen NJ, 2017).

Temos utilizado uma combinação de mucosectomia e ablação de possível displasia residual com eletrocoagulaçáo com plasma de argônio, com bons resultados (Machado G; Novaes H, 2001). Recentemente, o protocolo do Colégio Americano de Gastroenterologia (Shaheen NJ et al., 2016) recomenda uma combinação de mucosectomia e ablação por radiofrequência.

\section{Dissecçáo submucosa (ESD)}

Esta técnica, introduzida no Japão, é utilizada para a ressecção de lesões esofagianas extensas (Yamamoto $\mathrm{H}$ et al., 2002). A principal vantagem da dissecção endoscópica submucosa é que permite a retirada de lesóes "em bloco", porém o seu tempo de execuçáo é mais prolongado e o risco de complicaçóes é maior, se comparada com a mucosectomia (Bergmann JJ, Jul 11, 2018), como também parece não haver diferença significativa quanto à evolução clínica dos pacientes (Terheggen G et al., 2017).

Quanto às complicaçóes, estenose, perfuração e sangramento a longo prazo são as mais frequentes, embora raras em mãos experientes (Chevaux JB et al., 2015).

\section{ComentáRIos Finais E Conclusótes}

1. A incidência de adenocarcinoma do esôfago aumentou $500 \%$ desde 1970;

2. uma das condiçôes pré-malignas para o desenvolvimento do adenocarcinoma esofagiano, o esôfago de Barrett, tem risco anual para progredir para displasia de 
alto grau/adenocarcinoma estimado em 0,58\% - 0,63\% (Wani S et al., 2011);

3. o Barrett é assintomático em cerca de 6\% dos pacientes (Modiano N; Gerson LB, 2007);

4. é necessário o emprego de procedimentos especializados para o estabelecimento de seu diagnóstico definitivo - endoscopia digestiva alta e confirmação histopatológica (ASGE Standards of Practice Committee, 2012) - e o acesso à endoscopia está longe de ser universal, mesmo considerando os pacientes que apresentam sintomatologia;

5. a análise das endoscopias ou da histopatologia de hospitais qualificados náo representam a verdadeira prevalência do esôfago de Barrett;

6. os casos identificados por estes exames, provavelmente, diferem em termos de características demográficas e mesmo de saúde dos indivíduos que vivem com esôfago de Barrett não diagnosticado;

7. levando-se em conta estas limitaçóes, os dados epidemiológicos mostram que a doença do refluxo gastroesofagiano é um importante fator de risco para o desenvolvimento da metaplasia de Barrett, que ocorre em 6\% - 14\% destes pacientes;

8. tanto os métodos térmicos como a mucosectomia sáo efetivos para a erradicação do esôfago de Barrett;
9. estes métodos são de execução relativamente simples, do ponto de vista técnico;

10. o índice de complicaçóes é pequeno, a maioria das quais é leve;

11. os índices de morbidade e mortalidade são baixos;

12. Edwards et al. (1996) e Cameron \& Carpenter (1997) mostraram que o exame das peças cirúrgicas de $11 \%$ a $67 \%$ dos pacientes com diagnóstico prévio de displasia de alto grau, estabelecido em biópsias endoscópicas, já apresentavam adenocarcinoma e Konda et al. (2008) encontraram uma prevalência de $12 \%$, utilizando critérios estritamente histopatológicos de carcinoma invasivo.

Assim:

1. é melhor tratar os pacientes com displasia de baixo grau uma vez que, provavelmente, apresentaráo melhores resultados;

2. nesta eventualidade (displasia de baixo grau), os métodos de ablação e a mucosectomia são os que apresentam melhores resultados. Dentre eles, a ablação por radiofrequência e a eletrofulguração com plasma de argônio são aqueles que oferecem menor índice de complicações e melhor relação custo-benefício, sendo que o primeiro (ablação por radiofrequência) tem custo muito elevado, o que constitui importante limitaçáo para seu emprego em nosso País; 
3. para o adenocarcinoma associado ao esôfago de Barrett, a ressecção endoscópica (mucosectomia, dissecção da submucosa) é considerada a melhor opção terapêutica se a lesão estiver restrita à camada mucosa, porque estes tumores têm baixas taxas de metástases para os gânglios linfáticos (<3\%);

4. o consenso é menor em relação aos tumores que comprometem a muscular da mucosa, embora um número apreciável de serviços de endoscopia indique a ressecção endoscópica para estes pacientes (Bergmann JJ, Jul 11, 2018);

5. a esofagectomia está indicada para as lesôes que invadem a submucosa, considerando que essas lesóes cursam com taxas elevadas de metástases linfoganglionares (>25\%) (Alvarez-Herrero L, et al., 2010);

6. finalmente, ainda é necessário acumular maior casuística de pacientes acompanhados a longo prazo, para assegurar que estes métodos iráo melhorar a evolução clínica desta enfermidade e a consequente diminuição da incidência de adenocarcinoma do esôfago.

É necessário assinalar que ainda existem questóes à espera de respostas consensuais, como:

- Em relação às técnicas de ablação por via endoscópica:

Métodos isolados ou em combinação?
Qual é o melhor método de erradicação?

- Em relação à metaplasia intestinal residual após o tratamento endoscópico: É muito difícil ou mesmo impossível documentar a eliminação total das células caliciformes por meio de biópsias endoscópicas; por outro lado, o significado da metaplasia intestinal residual é ainda desconhecido.

- Em relação ao esôfago de Barrett sem displasia:

A ablação endoscópica, nestes casos, seria um "zelo excessivo"?

- $\quad$ Em relação à displasia de alto grau:

A terapêutica endoscópica seria um "tratamento insuficiente"?

- Em relação à escolha da melhor conduta terapêutica e à idade do paciente: Em jovens com baixo risco cirúrgico, as vantagens da esofagectomia seriam maiores que as desvantagens de um adenocarcinoma passar despercebido?

- Em relação ao câncer da junção esôfagogástrica:

Ainda não existem dados estatísticos suficientes para se demonstrar que a erradicação do epitélio metaplásico de Barrett e sua substituição por epitélio escamoso esofagiano normal resultaria em uma redução do número de casos de câncer da junção esôfago gástrica. 


\section{REFERÊNCIAS \\ Bibliográficas}

1. Pohl H, Welch HG. The role of overdiagnosis and reclassification in the marked increase of esophageal adenocarcinoma incidence. J Natl Cancer Inst 2005;97:142-6.

2. Wani S, Falk G, Hall M, et al. Patients with nondysplastic Barrett's esophagus have low risk for developing dysplasia or esophageal adenocarcinoma. Clin Gastroenterol Hepatol 2011;9:2207; quiz e26.

3. Barrett NR. The lower esophagus lined by columnar epithelium. Surgery 1957 ; 1:881- 894 .

4. Spechler SJ, Souza RF. Barrett's esopahgus. N Engl J Med 2014;371:931.

5. Spechler SJ. Barrett's esophagus: Pathogenesis and malignant transformation. UpToDate August 1, 2018.

6. Modiano N, Gerson LB. Barrett's esophagus incidence, etiology, pathophysiology, prevention and treatment. Thor Clin Risk Manag 2007;3: 1035-1145.

7. ASGE Standards of Practice Committee, Evans JA et al. The role of endoscopy in Barrett's esophagus and other premalignant conditions of the esophagus. Gastrointest Endosc 2012;76:1087.
8. Steevens J, Schouten LJ, Driessen AL et al. A prospective cohort study on overweight, smoking, alcohol consumption, and risk of Barrett's esophagus. Cancer Epidemiol Biomarkers Prev 2010.

9; Coleman HG, Bhat S, Johnston BT et al. Tobacco smoking increases the risk of high-grade dysplasia and cancer among patients with Barrett's esophagus. Gastroenterology 2012;142:233-40.

10. Hampel H, Abraham NS, El-Serag HB. Meta-analysis obesity and the risk for gastroesophageal reflux disease and its complications. Ann Intern Med 2005;143:199-211.

11. Wang K, Sampliner R. Updated guidelines 2008 for the diagnosis, surveillance and therapy of Barrett's esophagus. Am J Gastroenterol 2008;103:788-97.

12. Wild CP, Hardie LJ. Reflux, Barrett's oesophagus and adenocarcinoma: burning questions. Nat. Rev. Cancer 2003; 3: 676-84.

13. Spechler SJ, Sharma P, Souza RF, et al. American Gastroenterological Association medical position statement on the management of Barrett's esophagus. Gastroenterology 2011;140:1084-1091.

14. Spechler SJ, Sharma P, Souza RF, et al. American Gastroenterological Association medical position statement on the management of 
Barrett's esophagus. Gastroenterology 2011;140:e18-e52.

15. Prasad GA, Bansal A, Sharma P, et al. Predictors of progression in Barrett's esophagus: current knowledge and future directions. Am J Gastroenterol 2010;105:1490-1502.

16. Shaheen NJ, Falk GW, Iyer PG et al. ACG Clinical Guideline: Diagnosis and Management of Barrett's Esophagus. Am J Gastroenterol 2016;111:30.

17. Bennett C, Moayyedi P, Corley DA, et al. BOB CAT: A Large-Scale Review and Delphi Consensus for Management of Barrett's Esophagus With No Dysplasia, Indefinite for, or Low-Grade Dysplasia. Am J Gastroenterol 2015;110:662.

18. Bennett C, Vakil N, Bergman J, et al. Consensus Statements for Management of Barrett's Dysplasia and Early-Stage Esophageal Adenocarcinoma, Based on a Delphi Process. Gastroenterology 2012;143:336-46.

19. Berenson MM. Ablation therapy of Barrett's esophagus: measures of success and failure. Am J Gastroenterol 1998;93:1794-5.

20. Sampliner RE, Fennerty M, Garewal H. Reversal of Barrett's esophagus with acid suppression and multipolar electrocoagulation: preliminary results. Gastrointest Endosc 1996;44:532-35.

21. Bergman JJ. Barrett's esophagus: Treatment of high-grade dysplasia or early cancer with endoscopic resection. UpToDate 2018, July 11.

22. Sampliner RE. Ablation of Barrett's Mucosa. The Gastroenterologist 1997;5:185-88.

23. Prasad GA, Wang KK, Butter NS, et al. Long-term survival following endoscopic and surgical treatment of high-grade dysplasia in Barrett's esophagus. Gastroenterology 2007;132:1226-33.

24. Phoa KN, van Vilsteren FG, Weusten $\mathrm{BL}$, et al. Radiofrequency ablation vs endoscopic surveillance for patients with Barrett esophagus and low-grade dysplasia: a randomized clinical trial. JAMA 2014;311:1209-17.

25. Fitzgerald RC, di Pietro $M$, Ragunath K, et al. British Society of Gastroenterology guidelines on the diagnosis and management of Barrett's oesophagus. Gut 2014;63:7-42.

26. Hur C, Choi SE, Rubenstein JH, et al. The cost effectiveness of radiofrequency ablation for Barrett's esopahgus. Gastroenterology 2012;143:567-75.

27. Machado G. Endoscopia terapêutica em doenças esofagianas. In: GalvãoAlves J (ed). Temas de Atualização em Gastroenterologia. Grafitto Gráfica e Editora, Rio de Janeiro, 2016:43-65.

28. Fleischer DE. Comparing apples with apples and oranges: the role of radiofrequency ablation alone versus 
radiofrequency ablation plus EMR for endoscopic management of Barrett's esophagus with advanced neoplasia. Gastrointest Endosc 2012;76:740-42.

29. Singh S, Garg SK, Singh PP, et al. Acid-suppressive medications and risk of oesophageal adenocarcinoma in patients with Barrett's oesophagus: a systematic review and meta-analysis. Gut 2014;63:1229.

30. Van Laethem JL, Peny MO, Salmon I, et al. Intramucosal adenocarcinoma arising under squamous reepithelialisation of Barrett's oesophagus. Gut 2000;46:574.

31. Ghorbani S, Tsai FC, Greenwald BD, et al. Safety and efficacy of endoscopic spray cryotherapy for Barrett's dysplasia: results of the National Cryospray Registry. Dis Esophagus 2016;29:2417.

32. Spechler SJ. Barrett's esophagus: Surveillance and management. UpToDate August 3, 2018.

33. Machado G, Novaes H. Barrett's oesophagus: endoscopic thermal ablation of dysplasia. In: Marcon N, Haber G, Kortan P, Kandel GP (eds). 14th International Course on Therapeutic Endoscopy Syllabus, the Wellesley Group, St. Michael's Hospital, Toronto, Canada, October 2001, p.12-12.10.

34. Sharma VK, Wang KK, Overholt BE, et al. Balloon-based, circumferencial, endoscopic radiofrequency ablation of Barrett's esophagus: 1-year follow-up of 100 patients. Gastrointest Endosc 2007;65:185.

35. Sharma VK, Jae Kim H, Das A, et al. Circumferential and focal ablation of Barrett's esophagus containing dysplasia. Am J Gastroenterol 2009;104: 310-317.

36. Shaheen NJ, Sharma P, Overholt BF, et al. Radiofrequency ablation of Barrett's esophagus with dysplasia. N Engl J Med 2009;360:2277-88.

37. Orman ES, Li N, Shaheen NJ. Efficacy and durability o radiofrequency ablation for Bassett's esophagus: systematic review and meta-analysis. Clin Gastroenterol Hepatol 2013;11:1245.

38. Small AJ, Sutherland SE, Hightower JS, et al. Comparative risk of recurrence of dysplasia and carcinoma after endoluminal eradication therapy of high-grade dysplasia versus intramucosal carcinoma in Barrett's esophagus. Gastrointest Endosc 2015;81:1158.

39. Ell C, May A, Gossner L, et al. Endoscopic mucosal resection of early cancer and high-grade dysplasia in Barrett1s esophagus. Gastroenterology 2000;118:670.

40. Lewis JJ, Rubenstein JH, Singal AG, et al. Factors associated with esophageal 
stricture formation after endoscopic mucosal resection for neoplastic Barrett's esophagus. Gastrointest Endosc 2011;74:753.

41. Katada C, Muto M, Manabe T, et al. Esophageal stenosis after endoscopic mucosal resection of superficial esophageal lesions. Gastrointest Endosc 2003;57:165.

42. Chennat J, Konda VJ, Ross AS et al. Complete Barrett's eradication endoscopic mucosal resection: an effective treatment modality for highgrade dysplasia and intramucosal carcinoma-an American single-center experience. Am. J. Gastroenterol. 2009; 104: 2684-92.

43. Pech O, May A, Manner H, et al. Longterm efficacy and safety of endoscopic resection for patients with mucosal adenocarcinoma of the esophagus. Gastroenterology 2014;146:652.

44. Eluri S, Shaheen NJ. Barrett's esophagus: diagnosis and management. Gastrointest Endosc 2017;85:889-903.

45. Yamamoto $H$, Sekine $Y$, Higashizawa $T$, et al. A new technique for endoscopic mucosa resection with an insulatetip electrosurgical knife improves the completenesss of resection of intramucosal gastric neoplasms. Gastrointest Endosc 2002;54:629.

46. Terheggen G, Horn Em, Vieth M, et al. A randomised trial of endoscopic submucosal dissection versus endoscopic mucosal resection for early Barrett's neoplasia. Gut 2017;66:783.

47. Chevaux JB, Piessevaux H, JouretMourin A, et al. Clinical outcome in patients treated with endoscopic submucosal dissection for superficial Barrett's neoplasia. Endoscopy 2015;47:103.

48. Edwards MJ, Gable DR, Lentsch AB et al, The rationale for esophagectomy as the optimal therapy for Barrett's esophagus with high-grade dysplasia. Ann Surg. 1996;223:585-589.

49. Cameron A J, Carpenter H A Barrett's esophagus, high-grade dysplasia, and early adenocarcinoma: a pathological study. Am J Gastroenterol. 1997;92:586-591.

50. Alvarez-Herrero L, Pouw RE, van Vilsteren FG, et al. Risk of lymph node metastasis associated with deeper invasion by early adenocarcinoma of the esophagus and cardia: study based on endoscopic resection specimens. Endoscopy 2010;42:1030. 